\title{
WiP Abstract: A Physiology-Aware Communication Architecture for Distributed Emergency Medical CPS
}

\author{
Mohammad Hosseini \\ UIUC \\ shossen2@illinois.edu
}

\author{
Richard R. Berlin \\ Carle Foundation Hospital \\ richard.berlin@carle.com
}

\author{
Lui Sha \\ UIUC \\ lrs@illinois.edu
}

\begin{abstract}
For emergency medical cyber-physical systems, enhancing the safety and effectiveness of patient care, especially in rural areas, is essential. While the doctor to patient ratio in the United States is 30 to 10,000 in large metropolitan areas, it is only 5 to 10,000 in most rural areas; and the highest death rates are often found in the most rural counties [3]. Use of telecommunication technologies can enhance effectiveness and safety of emergency ambulance transport of patients from rural areas to a regional center hospital. It enables remote monitoring of patients by the physicians at the center hospital and provides vital assistance to the emergency medical technicians (EMT) to associate best treatments. However, the communication along the roads in rural areas can range from $4 \mathrm{G}$ to $2 \mathrm{G}$ to low speed satellite links, with some parts suffering from communication breakage. This unreliable and limited communication bandwidth together with the produced mass of clinical data and the many information exchanges pose a major challenge in real-time supervision of patients.

During this research, we are developing a novel adaptive physiology-aware communication architecture which is aware of the patient condition, the underlying network bandwidth, and the criticality of clinical data in the context of the specific disease to achieve an enhanced remote monitoring. Further, it features reliability, safety, and fault tolerance for cases such as network interruption.
\end{abstract}

\section{CCS CONCEPTS}

•Networks $\rightarrow$ Network architectures;

\section{KEYWORDS}

Physiology-aware communication, medical CPS, distributed models ACM Reference format:

Mohammad Hosseini, Richard R. Berlin, and Lui Sha. 2017. WiP Abstract: A Physiology-Aware Communication Architecture for Distributed Emergency Medical CPS. In Proceedings of The 8th ACM/IEEE International Conference on Cyber-Physical Systems, Pittsburgh, PA USA, April 2017 (ICCPS 2017), 1 pages. DOI: $10.1145 / 3055004.3064841$

\section{METHODOLOGY OVERVIEW}

The main goal of our research is to propose an adaptive modeldriven communication architecture for dynamic physiological systems that are distributed across a rural hospital, a tertiary center hospital, and an ambulance. The objective of our proposed physiology-aware communication architecture is to promote the smoothness and effectiveness of emergency patient care under such distributed settings. Using the concept of distributed model-driven physiological automata, we seek to exploit the semantics relation of clinical data communication, criticality of clinical data, and an

Permission to make digital or hard copies of all or part of this work for personal or classroom use is granted without fee provided that copies are not made or distributed for profit or commercial advantage and that copies bear this notice and the full citation on the first page. Copyrights for components of this work owned by others than ACM must be honored. Abstracting with credit is permitted. To copy otherwise, or republish, to post on servers or to redistribute to lists, requires prior specific permission and/or a fee. Request permissions from permissions@acm.org.

ICCPS 2017, Pittsburgh, PA USA

(C) 2017 ACM. 978-1-4503-4965-9/17/04 ..\$15.00

DOI: $10.1145 / 3055004.3064841$ ambulance's undertaken route in a disease-specific manner. In summary, the major challenges include, but are not limited to:

- Dynamic distribution of expertise, facilities, and physiological best-practice models among a rural hospital, an ambulance, and a center hospital

- Patient monitoring in ambulances under limited and variable bandwidth given the rural environment, and

- Communication breakage in parts of major routes, especially along rural routes.

To address the challenges above, we seek the following contributions throughout our research.

(1) Proposing the notion of distributed physiological bestpractice systems and to model them as communicating medical executable automata. Within this context, we propose model-driven message-exchange communication mechanisms to enable executable medical models to exchange messages with eachother so that their states get synchronized in a safe manner. We design a physiologyaware communication protocol that meets the reliability and safety requirements of emergency care with support of message prioritization. We partially implemented and evaluated our physiology-aware communication architecture over multiple executable medical models conducted in collaboration with Carle Foundation Hospital [1, 3, 4].

(2) Design of a bandwidth-compliant criticality-aware system for transmission of clinical data adaptive to varying bandwidths and patient's varying physiological states during ambulance transport in rural areas. Our adaptation techniques aim to transmit more critical clinical data with higher fidelity in response to changes in both bandwidth and patient condition. As a part of our contributions, we developed a bandwidth profiler in collaboration with Carle's ambulance service center, and used that to collect various geo-communication traces to support our experiments [2].

(3) Design of a disease-aware ambulance route scheduler integrated into our physiological communication architecture to assist ambulances pick the best route when continuous network coverage is needed for real-time remote monitoring of patients. Our novel physiological scheduler seeks trade-offs in travel time and availability of continuous network coverage during ambulance patient transport which aims to minimize a cost function through approximation of an NP-hard problem.

\section{REFERENCES}

[1] Mohammad Hosseini, Richard Berlin, Jiang Yu, and Lui Sha. 2016. ModelSink: A Middleware for Automatic Synchronization of Distributed Medical Models. ACM Transactions on Cyber-Physical Systems (ACM TCPS), submitted (2016).

[2] Mohammad Hosseini, Richard Berlin, Jiang Yu, and Lui Sha. 2017. BandwidthCompliant Criticality-Aware Clinical Data Communication in Ambulances. IEEE Transactions on Multimedia (IEEE TMM), submitted (2017).

[3] Mohammad Hosseini and et al. 2016. A Pathophysiological Model-Driven Communication for Dynamic Distributed Medical Best Practice Guidance Systems. Fournal of Medical Systems (2016).

[4] Mohammad Hosseini, Yu Jiang, Poliang Wu, Richard B. Berlin, Jr., and Lui Sha. 2015. SINk: A Middleware for Synchronization of Heterogeneous Software Interfaces. In Proceedings of the 14th International Workshop on Adaptive and Reflective Middleware (ARM 2015). ACM, 2:1-2:6. 